\title{
A Two Step Gaussian Modelling to Assess PPG Morphological Variability Induced by Psychological Stress
}

\author{
Swati Banerjee ${ }^{1}$, Raquel Bailón ${ }^{1}$, Jesus Lazáro ${ }^{2}$, Vaidotas Marozas ${ }^{3}$, Pablo Laguna $^{1}$, Eduardo Gil ${ }^{1}$ \\ ${ }^{1}$ BSICoS, Aragón Institute for Engineering Research (I3A), IIS Aragón, University of Zaragoza \\ (UZ), Spain, and Biomedical Research Networking center in Bioengineering, Biomaterials and \\ Nanomedicine (CIBER-BBN), Madrid, Spain \\ ${ }^{2}$ Department of Electrical Engineering (ESAT), STADIUS Centre for Dynamical Systems, Signal \\ Processing and Data Analytics, KU Leuven, Leuven, Belgium IMEC, Leuven, Belgium \\ ${ }^{3}$ Biomedical Engineering Institute, Kaunas University of Technology, Kaunas, Lithuania
}

\begin{abstract}
In the present work, we analyse characteristic features of the pulses photoplethysmographic (PPG) signal and their changes and evolution during various psycologically stressed conditions. Acute psychological stress was induced to subjects using a modified version of Trier Social Stress Test. It was revealed that the morphology of the $P P G$ pulses is different during the streneous sessions from that of the relaxed one and possibly reflect arterial stiffness variations which can be a marker for differentiation of the stressed stages from the relaxed stage of the protocol. Since, the PPG pulse formation is due to the superimposition of a systolic and a reflected wave, a novel two step Gaussian mixture modelling technique was used to extract their relative arrival time and width. Position $\left(B_{1}, B_{2}\right)$ and width $\left(C_{1}, C_{2}\right)$ of the systolic and reflected wave are the relevant features studied along with the time to reflection $\left(T_{12}\right)$. Significant statistical differences were observed in $B_{1}(p<0.001), B_{2}(p<0.05), C_{1}(p<0.001), C_{2}(p<0.05)$ in the relaxed and stressed session. These results suggest changes in the morphology of PPG pulses possibly revealing marks of arterial stiffness occurring due to psychological stress.
\end{abstract}

\section{Introduction}

Stress is considered as the 'world health epidemic' of the $21^{s t}$ century by the World Health Organisation and is perceived as a serious health condition [1]. Stress is actually the response of an organism to harmful situations or to a hostile environment that the individual perceives as a 'threat' [2]. The Autonomic Nervous System (ANS), has a direct function in the physical response to stress and is divided into the Sympathetic Nervous System (SNS), responsible for reflexes for perceived danger and Parasym- pathetic nervous system, responsible for the 'rest and digest' phenomenon. The ANS signals the adrenal glands to release hormones called adrenalin and cortisol responsible for changes in heart rate, respiration rate, stiffness of arteries, digestive process to change and the glucose level in the bloodstream to increase for dealing with the currently perceived emotional emergency [3]. Chronic stress aggravate risk of cardiovascular diseases, hypertension, myocardial infarction, strokes and diabetes and can eventually become the cause of sudden death [5].

Proper screening and monitoring of psychological stress has become an essential requirement and requires a reliable non-invasive tool for the continuous estimation of the stress level. The goal of ES3 [6] project is to develop an efficient tool for the estimation of stress which includes different physiological signals, biochemical markers and psychometric questionnaires, during physical, emotional and even chronic stress. In one of the pilot studies within the ES3 project, acute psychological stress was induced to healthy young volenteers using Trier social Stress Test (TSST) [10]-[11]. Each task has different demanding conditions generating stress in the subject. A previous study showed that respiratory frequency and heart rate variability analysis, when guided by respiration, could differentiate stress from relaxed stages of the protocol [7].

There are evidences in research that acute mental stress has a prolonged unfavorable effect on arterial stiffness and wave reflections which can be estimated from the changes in the morphology of the Photoplethsmography (PPG) signal [8]. PPG is a non-invasive technique commonly applied for monitoring of hemodynamic changes in the cardiovascular system by illuminating tissue with a certain wavelength and measuring the transmitted or reflected light, which is even simpler to acquire than the ECG. PPG based analysis technique has been used for arrhythmia detection and detection of premature beats. 
One pulse of a PPG signal is composed of a systolic wave and a diastolic or reflected wave. The PPG pulse amplitude is related to ventricular filling and along with the relative position of the wave occurrance with respect to the QRS complex of ECG, can give vital indication of the changing homeostasis of the body condition under stress.

The morphological variations of the PPG pulses occuring due to arterial stiffness among the relaxing and stressful sessions of the protocol are studied using a novel two step Gaussian mixture Modelling. The studied morphological features are the amplitudes $\left(A_{1}, A_{2}\right)$, pulse wave arrival time $\left(B_{1}, B_{2}\right)$, widths $\left(C_{1}, C_{2}\right)$ of the systolic and reflected wave respectively, as well as the reflection index $R I\left(A_{1} / A_{2}\right)$ and time to reflection $T_{12}\left(B_{2}-B_{1}\right)$.

\section{Material and method}

\subsection{Protocol setup and data collection}

A database containing records from 25 volunteers ( 9 men and 16 women) in an age range of $21.56 \pm 2.71$ years was acquired in the University of Zaragoza (UZ). The protocol was approved by the Ethics Committee at the UZ [6]. The basal session $\left(B L_{R}\right)$ consisted of a 35-minutes-length relaxing audition. The stress session tries to induce emotional stress by a modified version of the Trier Social Stress Test [8]. This session included the following stages:

- Baseline stage during stress session $\left(B L_{S}\right)$ : 10-minutes length relaxing audition.

- Story telling stage $(S T)$ : 3 stories are told to the subject with a great amount of details requiring a lot of attention to remember.

- Memory task $(M T)$ : the subject is requested to tell back every remembered detail within 30 seconds for each story. - Stress anticipation (SA): subject is requested to wait for the evaluation of the memory test. The duration of this stage is 10 minutes.

- Video Display $(V D)$ : a projection of a video with the subject performance in the memory test is shown.

- Arithmetic Task $(A T)$ : the subject has to count down from 1022 in steps of 13. In case of a calculation error, they were requested to restart from 1022 again. Although it is not expected to complete the countdown within 5 minutes, all the subjects were requested to do so to create an additional mental load. The last five stages are considered stressful. $B L_{B}, B L_{S}$ and $S A$ have longer duration than that of the other stages. The arithmetic task was assumed to be the most stressful of all as of the task due to it's nature and also because it is performed at the end of all the other stressful sessions. A detailed description of the protocol is presented in [7]. A pictorial overview of the protocol is depicted in Figure 1.

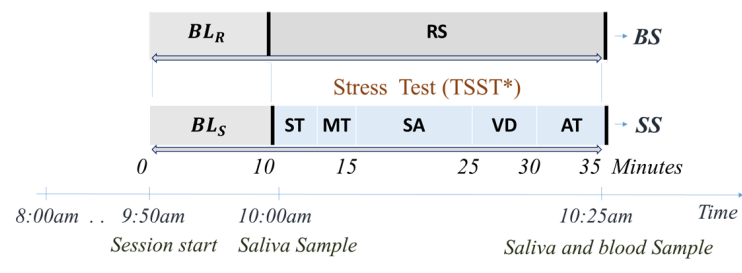

Figure 1. Stages of the Protocol

\subsection{PPG signal preprocessing and pulse segmentation}

The PPG signal was denoised using a zero phase lowpass butterworth filter with a cut off frequency of $20 \mathrm{~Hz}$. To understand the underlying homeostasis of the subject under acute psychological stress and study the corresponding changes in the morphology of the PPG pulses one cardiac cycle was considered for analysis. Individual PPG pulses were segmented using a PPG basal point detecter developed in [12]. As the time duration of the pulse varies with the heart rate they were normalized/rescaled temporally to 1000 samples for homogenity of analysis. Also their amplitude were normalized to unit. The pulses distorted with noise are rejected and not consider for analysis using a covariance based outlier rejection method.

\subsection{Two step Gaussian mixture model Fit- ting and physiological feature}

A mathematical model based on the sum of two Gaussians was used for modelling the PPG pulse for extraction of the characteristic features. Since the pulses in the original signals are due to the superimposition of a forward (systolic) and a backward wave (reflected), we use a model consisting of two primary Gaussian (1):

$$
y(n)=A_{1}^{r} e^{\frac{-\left(n-B_{1}^{r}\right)^{2}}{C_{1}^{r 2}}}+A_{2}^{r} e^{\frac{-\left(n-B_{2}^{r}\right)^{2}}{C_{2}^{r 2}}}
$$

Where the model parameters are:

- $\mathrm{A}_{1}^{r}, \mathrm{~A}_{2}^{r}$ : These are the amplitude of the systolic and the reflected wave respectively.

- $\mathrm{B}_{1}^{r}, \mathrm{~B}_{2}^{r}$ : Position of the systolic and the reflected wave respectively

- $\mathrm{C}_{1}^{r}, \mathrm{C}_{2}^{r}$ : Width of the systolic and the reflected wave respectively.

- $\mathrm{RI}^{r}$ (Reflection Index): $\mathrm{A}_{1}^{r} / \mathrm{A}_{2}^{r}$

- $\mathrm{T}_{12}^{r}$ (Time to reflection): $\mathrm{B}_{2}^{r}-\mathrm{B}_{1}^{r}$

The estimated features are in samples from the time normalized pulses or say the rescaled features (denoted with the superscript " $r$ ") for better comparison of the pulses.

Algorithm 1 presents the details of the calculations involved in estimation of the model (1) and then reverting back to the original time domain. To better capture the 
Table 1. Median \pm MAD for the Parameters for different Stages of the Protocol (in Sec)

\begin{tabular}{|c|c|c|c|c|c|c|}
\hline Parameter/Median & $\mathrm{BL}_{s}$ & $\mathrm{ST}$ & $\mathrm{MT}$ & $\mathrm{SA}$ & $\mathrm{VD}$ & $\mathrm{AT}$ \\
\hline$A_{1}$ & $7.3714 \pm 0.1069$ & $3.6928 \pm 0.1024$ & $3.2707 \pm 0.349$ & $5.3197 \pm 0.129$ & $3.1483 \pm 0.126$ & $1.1597 \pm 0.510$ \\
\hline$A_{2}$ & $4.9071 \pm 0.1837$ & $2.3063 \pm 0.0210$ & $2.0411 \pm 0.130$ & $3.8082 \pm 0.130$ & $1.9509 \pm 0.129$ & $0.6789 \pm 0.09508$ \\
\hline$B_{1}(\mathrm{sec})$ & $0.1321 \pm 0.0012$ & $0.1053 \pm 0.002^{*}$ & $0.1506 \pm 0.006^{*}$ & $0.1693 \pm 0.035^{*}$ & $0.2317 \pm 0.049^{* *}$ & $0.2068 \pm 0.098^{* *}$ \\
\hline$B_{2}(\mathrm{sec})$ & $0.3832 \pm 0.012$ & $0.3025 \pm 0.009^{*}$ & $0.3776 \pm 0.0039$ & $0.445 \pm 0.063^{*}$ & $0.503 \pm 0.014^{*}$ & $0.4560 \pm 0.040^{*}$ \\
\hline$C_{1}(\mathrm{sec})$ & $0.0740 \pm 0.0042$ & $0.0561 \pm 0.006$ & $0.0948 \pm 0.0124^{*}$ & $0.1125 \pm 0.033^{*}$ & $0.1475 \pm 0.0002^{*}$ & $0.1493 \pm 0.030^{* *}$ \\
\hline$C_{2}(\mathrm{sec})$ & $0.1978 \pm 0.0072$ & $0.1628 \pm 0.0013$ & $0.1574 \pm 0.0105^{*}$ & $0.1968 \pm 0.030$ & $0.179 \pm 0.0019^{*}$ & $0.1361 \pm 0.017^{* *}$ \\
\hline$T_{12}(\mathrm{sec})$ & $0.2371 \pm .0084$ & $0.2126 \pm 0.017^{*}$ & $0.2312 \pm 0.023$ & $0.2757 \pm 0.0162^{* *}$ & $0.298 \pm 0.016^{* *}$ & $0.247 \pm 0.0066^{*}$ \\
\hline
\end{tabular}

relevant features, it was proposed to perform the Gaussian fitting in two steps. We assume that the systolic pulse is the unique contributor to the uprising part of the PPG, and consequently we restrict the fitting of the systolic pulse to this part, to avoid the reflected pulse influencing the systolic estimation. Let, $p(n)$ be the amplitude and time normalised pulse under analysis and the first approximate systolic wave be represented by $s(n)$. Algorithm 1 gives the two step Gaussian mixture model.

Algorithm 1 : Two step Gaussian modelling of PPG pulses Step1: Estimation of the approximate systolic wave $s(n)$, Let, $p(n)$ be one PPG pulse to be studied, where $\mathrm{n}=1 \ldots 1000$

$p(\max )=\max \{p(n)\} ;$ Now, the approximate systolic wave is given by,

$s(n)=[p(1), \ldots, p(\max ), p(\max -1), \ldots, p(1)]$;

Fit, $s(n)$ with one Gaussian, $f_{1}(n)$ and estimating $\mathrm{A}_{1}^{r}$, $\mathrm{B}_{1}^{r}$, $\mathrm{C}_{1}^{r}$, Once, fitted it gives a proper representation of the physiologically relevant signatures of the systolic part of the pulse

Step 2: Estimation of the residual $e(n)$,

$e(n)=p(n)-f_{1}(n)$, fitting with Gaussian $f_{2}(n)$ for estimating parameters $\mathrm{A}_{2}^{r}, \mathrm{~B}_{2}^{r}$ and $\mathrm{C}_{2}^{r}$

Computing the parameters $\mathrm{RI}^{r}, \mathrm{~T}_{12}^{r}$. Scaling back the parameters obtained, $A_{1}, A_{2}, B_{1}, B_{2}, C_{1}, C_{2}, R I, T_{12}$ to their original time and amplitude for physiological relevance using appropriate scaling factors.

The sum of the Gaussian $f(n)=f_{1}(n)+f_{2}(n)$ is now the representation of the pulse $p(n)$ with an error factor. However, this suits the goal of the study as it was intended to estimate the primary systolic and the reflected waves properties and identify the related changes in various stages of the protocol to differentiate between stress stages from that of the relaxed stages.

For the physiological relevance the estimated parameters requires to be scaled back to their original time, we use the following scaling factors:

- $A_{1}=A_{1}^{r} \cdot h ; A_{2}=A_{2}^{r} \cdot h$

- $B_{1}=B_{1}^{r} w /\left(1000 \cdot f_{s}\right) ; B_{2}=B_{2}^{r} w /\left(1000 \cdot f_{s}\right)$

- $C_{1}=C_{1}^{r} w /\left(1000 \cdot f_{s}\right) ; C_{2}=C_{2}^{r} w /\left(1000 \cdot f_{s}\right)$

- $R I$ (Reflection Index) $=A_{1} / A_{2}$

- $T_{12}$ (Time to reflection) $=B_{2}-B_{1}$ where, $w$ is the original width in samples before resampling to 1000 samples, $h$ is the actual height of the pulse and $f_{s}$ is the sampling rate.

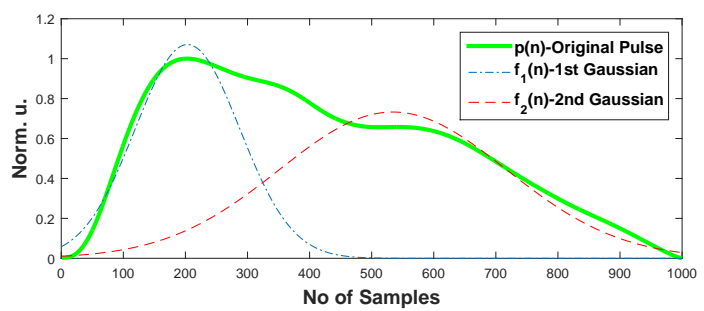

Figure 2. Pulse decomposition method for Stress Prerelaxed Session

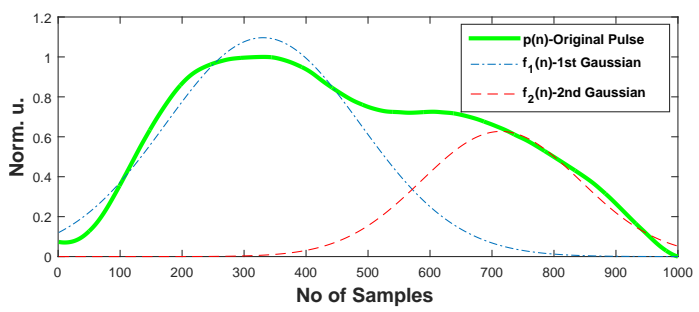

Figure 3. Pulse decomposition method for Stress Arithmetic Session

\subsection{Statistical Analysis}

Once the parameters are extracted they were analysed for statistical significance using Wilcoxon test, where only ( $p$ value $<0.05^{*}$ and $p$ value $<0.001^{* *}$ ) are considered as significantly different for identification of the stress stages of the protocol. Table 1 shows the overall results in terms of median and the median absolute deviation (MAD) of the dataset. The values are in second and are scaled back to match the temporal characteristic of the original pulses.

\section{Results and Discussion}

Fig. 2 and Fig. 3 show an example of the two step gaussian model fitting of a pulse from $B L_{s}$ and an $A T$ session respectively. It was revealed that the features $B_{1}, B_{2}, C_{1}, C_{2}$ 
and $T_{12}$ are statistically significant for further classification of the stressing stages from the $B L_{s}$ stage. It was observed that the values for $\mathrm{B}_{1}, \mathrm{~B}_{2}, \mathrm{C}_{1}, \mathrm{C}_{2}$ are very different between the $B L_{S}$ and $A T$ sessions of the protocol. Analyzing this differences we see that $C_{2}$ (width of the reflected pulse) is narrowed at stress stage, as can be hypothesized from a stiffer vessel provoking higher pulse speed and so lower width. The reverse is observed with $C_{1}$ and $T_{12}$, which do not follow the mentioned hypothesis. $A_{1}, A_{2}$ and $R I$ are not considered for analysis in this manuscript because the two Gaussian functions $f_{1}(n)$ and $f_{2}(n)$ does not exctly coincide with the systolic and the reflected part of the PPG pulses. This can be due to the fact that more than two main pulses are the components of PPG [?] and hence claiming for more detailed modeling using more than two Gaussians to account for the extra pulse which can be disturbing the two pulse based modeling presented here.

Also, from an earlier study it has already been revealed that respiration and HRV indices are significantly changed during these stages showing the sympathetic activation. Future scope of this study includes classification of the subjects based on a scoring pattern once the stressed stages are being identified. Also we plan to include the biochemical markers acquired during the recording of the physiological signals.

\section{Conclusion}

Present analysis reveals that the characteristic features obtained from the morphology of the PPG pulses, changes from various stages among the protocol and is very different in the Arithmetic task session. This may be due to the fact that $A T$ is the most demanding stage of the protocol because of the nature of the task itself and also because the stage is the last of all the stress sessions. These results are possible indicator of arterial stiffness marks expressed through the changes in the morphology of the PPG pulses induced due to psychological stress.

\section{Acknowledgements}

This work is supported by project TIN2014-53567-R of UZ, funded by MINECO, by Biomedical Research Networking Center on Bioengineering, Biomaterials and Nanomedicine (CIBER-BBN) of the Institute of Health Carlos III, (ISCIII), by Grupo Consolidado BSICoS from DGA (Aragón) and European Social Fund (EU), and by UZ by project UZ2014-TEC-01.

\section{References}

[1] S. Leka, A. Griffiths, T. Cox, "Work organization and stress", Protecting Workers Health Series No. 3. Geneva: World Health Organization Library; 2003.
[2] H. Selye, "Stress and the General Adaptation Syndrome". British Medical Journal, vol. 1, no. 4667, pp. 1383 - 1392, 1950.

[3] R. Glaser and J. Kiecolt-Glaser, "Stress-induced immune dysfunction: implications for health", Nature Reviews Immunology, vol. 5, pp. 243-251, 2005.

[4] J. Hellhammer, M. Schubert, "The physiological response to Trier Social Stress Test relates to subjective measures of stress during but not before or after the test", Psychoneuroendocrinology, vol. 37(1), pp. 119124, 2012.

[5] K. L. Tamashiro, R.R Sakai, C.A Shively, I. N Karatsoreos and L. P Reagan, "Chronic stress, metabolism and metabolic syndrome", Stress, vol. 531 14, no. 5, pp. 468474, 2011.

[6] J. Aguilo, P. Ferrer-Salvans, A. Garcia-Rozo, A. Armario, A. Corbi, F. J. Cambra, R. Bailon, A. Gonzalez-Marcos, G. Caja, S. Aguilo, R. Lopez Anton, A. Arza-Valdes, J. M. Garzon-Rey, "Project ES3: attempting to quantify and measure the level of stress", Revista de neurologia, vol. 61, no. 9, 534 pp. 405-415, Nov. 2015.

[7] A. Hernando, J. Lazaro, E. Gil, A. Arza Valdes, J. GarzonRey, R. Lopez-Anton, C. de la Camara, P. Laguna, J. Aguilo, and R. Bailon, "Inclusion of Respiratory Frequency Information in Heart Rate Variability Analysis for Stress Assessment," IEEE journal of Journal of Biomedical and Health Informatics,vol. 20, no. 4, pp. 1016-1025, July 2016.

[8] C. Vlachopoulos, F. Kosmopoulo, N. Alexopoulos, N. Iokeimidis, G. Siasos, C. Stefanadis, "Acute Mental Stress Has a Prolonged Unfavorable Effect on Arterial Stiffness and Wave Reflections". Psychosomatic Medicine, vol. 68, no. 2, pp.231-237, March 2006.

[9] A. Solosenko, A. Petrenas, V. Marozas, "Photoplethysmography-based method for automatic detection of premature ventricular contractions", IEEE Transaction on Biomedical Circuits Systems, Vol 9, no. 5, pp. 662-669, 2015.

[10] S. Cohen, T. Kamarck, and R. Mermelstein, "A Global Measure of Perceived Stress", J. Health Soc. Behav., vol. 24, no. 4, pp. 385-396, 1983.

[11] C. D. Spielberger, R. L. Gorsuch, R. Lushene, P. R. Vagg, and G. A. Jacobs, "Manual for the State-Trait Anxiety Inventory", Palo Alto, CA: Consulting Psychologists Press, 1983.

[12] J. Lazaro, E. Gil, J. M. Vergara, and P. Laguna,"Pulse Rate Variability Analysis for Discrimination of SleepApnea-Related Decreases in the Amplitude Fluctuations of Pulse PhotoplethysmographicSignal in Children", IEEE Trans.Biomed. And Health Informatics, vol. 18, no. 1, pp. 1005-1014, Jan. 2014.

Address for correspondence:

Name: Swati Banerjee

Full postal address: Dept. Ingeniería Electrónica y Comunicaciones (IEC), Univ. de Zaragoza, c/Maria de luna 1, 50018 Zaragoza, Spain

E-mail address: swatibanerjee@ieee.org, swatibanerjee29@yahoo.com 